\title{
When are global units norms of units?
}

\author{
by \\ DAVID FOLK (Ypsilanti, Mich.)
}

1. Introduction. Let $L / K$ be a Galois extension of number fields, $\omega \in$ $K, H$ the Hilbert class field of $L$. Often it is possible to determine that $\omega=N_{L / K}(z)$, for some $z \in L$, while number theoretical questions often demand more: If $\omega$ is an element of $\mathcal{O}_{K}^{*}$, is it also the norm of an element $z \in \mathcal{O}_{L}^{*}$ ? In this paper, we prove that if $\omega$ is not the norm of a unit, then it is not even a global norm from $H$.

Throughout the paper, we will be using the following notation: For an arbitrary number field $M, \mathcal{O}_{M}$ will denote the ring of algebraic integers in $M, \mathcal{O}_{M}^{*}$ the group of units of $\mathcal{O}_{M}, P_{M}$ the principal ideals of $M$. Further, $A_{M}$ will denote the adeles of $M, A_{M}^{*}$ the group of ideles of $M$, with $I_{M}$ denoting the ideals of $M$. Finally, $C_{M}$ and $C l_{M}$ will denote the idèle class group of $M$ and the ideal class group of $M$, respectively.

TheOREM 1. Let $L / K$ be a Galois extension of number fields with $H$ the Hilbert class field of $L$. Then

$$
N_{H / K}\left(H^{*}\right) \cap \mathcal{O}_{K}^{*} \subset N_{L / K}\left(\mathcal{O}_{L}^{*}\right) .
$$

Proof. Let

$$
G:=\operatorname{Gal}(L / K) \quad \text { and } \quad \Gamma:=\operatorname{Gal}(H / K),
$$

with $\omega \in N_{H / K}\left(H^{*}\right) \cap \mathcal{O}_{K}^{*}$. We will also write $\omega$ for its image in the Tate cohomology group $\widehat{H}^{0}\left(\Gamma, \mathcal{O}_{H}^{*}\right)$. This is harmless, since we want to prove that $\omega \in N_{L / K}\left(\mathcal{O}_{L}^{*}\right)$ and the other representatives of the class of $\omega$ in $\widehat{H}^{0}\left(\Gamma, \mathcal{O}_{H}^{*}\right)$ differ from $\omega$ by an element of $N_{H / K}\left(\mathcal{O}_{H}^{*}\right) \subset N_{L / K}\left(\mathcal{O}_{L}^{*}\right)$. Consider the commutative diagram of $\Gamma$-modules, with exact rows and exact columns:

1991 Mathematics Subject Classification: 11R37. 


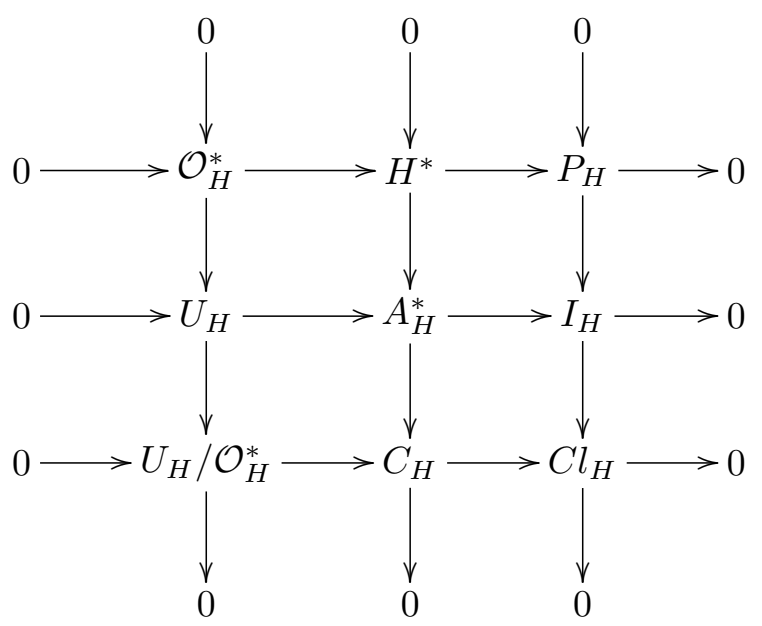

We thus derive the following diagram of $\Gamma$-cohomology groups:

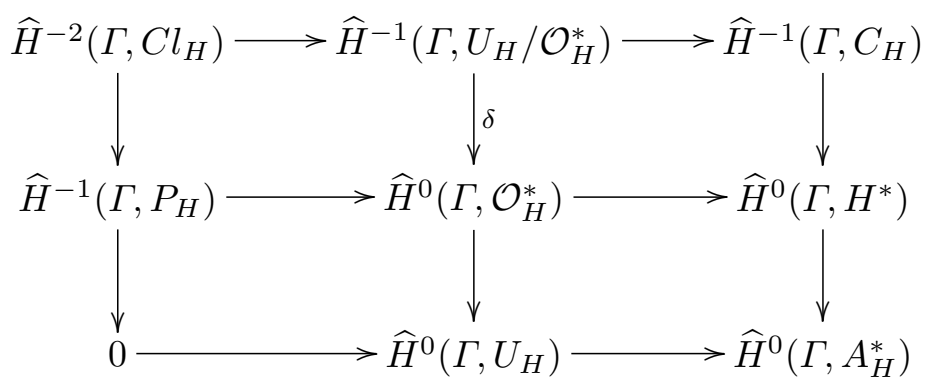

The zero in the lower left corner comes from the fact that $\widehat{H}^{-1}\left(\Gamma, I_{H}\right)=0$, which follows from Shapiro's lemma (see [2], Lemma 2.11 for details).

We now do a diagram chase: $\omega \in \widehat{H}^{0}\left(\Gamma, \mathcal{O}_{H}^{*}\right)$ maps to 0 in $\widehat{H}^{0}\left(\Gamma, H^{*}\right)$. Therefore it "comes from" $z \in \widehat{H}^{-1}\left(\Gamma, P_{H}\right)$. Because of the zero in the left lower corner, $z$ itself "comes from" $y \in \widehat{H}^{-2}\left(\Gamma, C l_{H}\right)$. Let $x$ denote the image of $y$ in $\widehat{H}^{-1}\left(\Gamma, U_{H} / \mathcal{O}_{H}^{*}\right)$. Let $x \in U_{H}$ also denote any unit idèle whose class in $\widehat{H}^{-1}\left(\Gamma, U_{H} / \mathcal{O}_{H}^{*}\right)$ is our $x$. By the exactness of the diagram, the class $x$ maps to 0 in $\widehat{H}^{-1}\left(\Gamma, C_{H}\right)$, hence the element $x \in H^{*} \cdot\left(A_{H}^{*}\right)^{I_{\Gamma}}$, where $I_{\Gamma}$ denotes the augmentation ideal of $Z[\Gamma]$, and $H^{*} \cdot\left(A_{H}^{*}\right)^{I_{\Gamma}}$ denotes the subgroup of $A_{H}^{*}$ generated by the set $\left\{x^{1-\sigma}: x \in A_{H}^{*}, \sigma \in \Gamma\right\}$ and $H^{*}$. Moreover, by the commutativity of the diagram, the map labeled $\delta$ maps the class $x$ to the class of $\omega$ in $\widehat{H}^{0}\left(\Gamma, \mathcal{O}_{H}^{*}\right)$. Recall the definition of the coboundary map $\delta: \widehat{H}^{-1}\left(\Gamma, U_{H} / \mathcal{O}_{H}^{*}\right) \rightarrow \widehat{H}^{0}\left(\Gamma, \mathcal{O}_{H}^{*}\right)$ : Choose any element $x \in U_{H}$ representing the class $x \in \widehat{H}^{-1}\left(\Gamma, U_{H} / \mathcal{O}_{H}^{*}\right)$; then $\delta(x)=$ the class of $N_{H / K}(x)$ in $\widehat{H}^{0}\left(\Gamma, \mathcal{O}_{H}^{*}\right) \cong \mathcal{O}_{K}^{*} / N_{H / K}\left(\mathcal{O}_{H}^{*}\right)$. Thus we see that the class of the

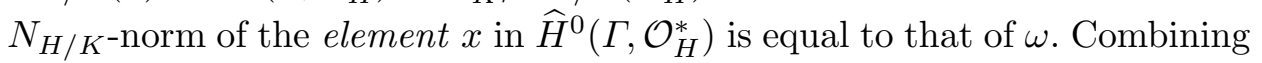


this with the fact that $\widehat{H}^{-1}\left(\Gamma, U_{H} / \mathcal{O}_{H}^{*}\right)$ is a submodule of $U_{H} /\left(U_{H}\right)^{I_{\Gamma}} \mathcal{O}_{H}^{*}$, we see that we may modify the element $x \in U_{H}$ by an element of $\mathcal{O}_{H}^{*}$ so that the elements $x$ and $\omega$ satisfy $N_{H / K}(x)=\omega$.

Let therefore $\alpha \in H^{*}$ such that $x \alpha^{-1} \in\left(A_{H}^{*}\right)^{I_{\Gamma}}$. Then

$$
\begin{aligned}
N_{H / L}\left(x \alpha^{-1}\right) \in N_{H / L}\left(\left(A_{H}^{*}\right)^{I_{\Gamma}}\right) & =\left(N_{H / L}\left(A_{H}^{*}\right)\right)^{I_{G}} \\
& \subset\left(U_{L} \cdot L^{*}\right)^{I_{G}}=U_{L}^{I_{G}} \cdot\left(L^{*}\right)^{I_{G}} .
\end{aligned}
$$

The first equality follows from the fact that $N_{H / L}$ is a central element in the group ring $Z[\Gamma]$, the inclusion from the fact that $H$ is the Hilbert class field of $L$ and the last equality is a generality. By $I_{G}$ we denote the augmentation ideal of the group $\operatorname{ring} Z[G]$.

So, we have $N_{H / L}\left(x \alpha^{-1}\right)=u \beta$, where $u \in U_{L}^{I_{G}}$ and $\beta \in\left(L^{*}\right)^{I_{G}}$. This implies that

$$
\eta=N_{H / L}(x) \cdot u^{-1}=N_{H / L}(\alpha) \cdot \beta \in L^{*} \cap U_{L}=\mathcal{O}_{L}^{*} .
$$

Since $N_{L / K}(u)=1$, we have $N_{L / K}(\eta)=N_{H / K}(x)=\omega$, as required.

I would like to express my deep appreciation to David Leep and Renè Schoof for their willingness to listen and for their wonderful suggestions.

\section{References}

[1] J. W. S. Cassels and A. Fröhlich (eds.), Algebraic Number Theory, Academic Press, New York, 1967.

[2] A. Fröhlich, Central Extensions, Galois Groups, and Ideal Class Groups of Number Fields, American Mathematical Society, 1980.

Department of Mathematics

Eastern Michigan University

Ypsilanti, Michigan 48197

U.S.A.

E-mail: mth-folk@online.emich.edu 